\title{
Multi Induction Motor Synchronous Drive System Based on Diagonal Recurrent Neural Network Control
}

\author{
Chong Chen*, Simin Peng, Zhilei Yao and Quanyu Wang \\ School of Electrical Engineering, Yancheng Institute of Technology \\ Yancheng 224051 PR China \\ cc082120@126.com
}

\begin{abstract}
Through the analysis of the mathematical model of multi induction motor synchronous drive system, a new control method based on neural network is proposed and applied into the system.The neural network controller is composed of self-tuning PID controller based on diagonal recurrent neural network (DRNN) and neuron decoupling compensator. The three self-tuning PID controllers are used in the speed and tension control loops respectively, making the system possess stronger adaptive capability and robustness. The neuron decoupling compensator integrates the effect of the three loops and realizes the adaptive decoupling control between speed and tension by training the weights of network to compensate the coupling relation. The experiment results show that, compared with traditional PID control, the control system can obtain optimal parameters according to online identification of the DRNN network, realizing the better decoupling control of speed and tension, and the system has better dynamic, static characteristics and robustness.
\end{abstract}

Keywords: neural network; DRNN; decoupling control; synchronous control; multi induction motor system

\section{Introduction}

Since controlled objects are more and more complex, the design theory for traditional control system is facing with severe challenges. Especially, as a complex industrial controlled object, there is coupling effect between each variable [1]. If the multi variable controller is designed with common method, as for industrial process, the linear constant and high precision mathematical model is difficult to achieve. Even if achieved, it is also difficult to meet the real industrial control requirements because of its complexity.

Artificial neural network has strong self-learning, adaptive and nonlinear mapping capability. When it is used to design control system, we only need to train the neural network online or offline, and then use the training results without the mathematical model of controlled object. If neural network control method is adopted to control the nonlinear and uncertain system, it will has strong adaptability and robustness. Therefore, artificial neural network can make up for the deficiency of common methods, making the decoupling control of multi variable system possible [2]-[3].

Aiming at the multi induction motor synchronous drive system working under constant $\mathrm{V}$ to $\mathrm{F}$, a new neural network compound controller composed of neuron decoupling compensator and self-tuning PID controller is presented in this paper [4]. Based on S7-300 experimental platform, many experiments are carried out and results show that the control system can get optimal parameters according to online identification of the DRNN network, and it realizes the better decoupling control of 
speed and tension with better performances of dynamic and static status. Thus, the method presented in this paper meets the requirements of many industrial control environments, with good application prospects.

\section{Mathematical Model of Multi Induction Motor Synchronous Drive System}

The physical model of multi induction motor synchronous drive system is shown in Figure 1. The 1\#motor controls line speed, while 2\#motor and 3\#motor perform the tension control $\mathrm{F}^{*}$. According to the huke laws, the basic dynamic equations can be derived by Eq. (1).In this paper, the tension is proportional to the speed difference between the adjacent two motors in the system [5]-[6].

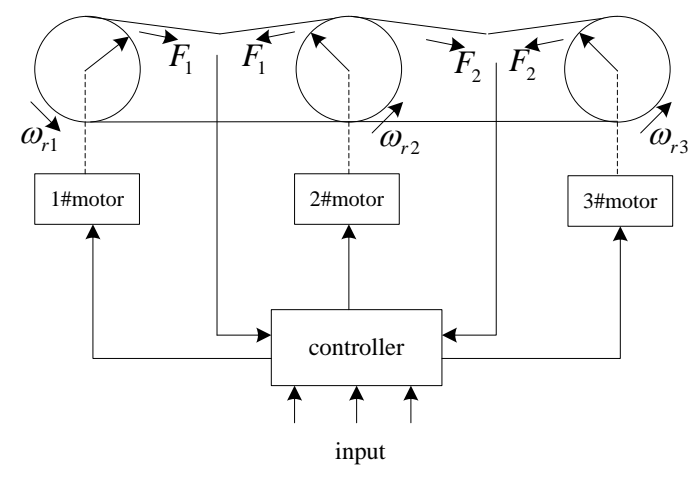

Figure 1. Three Motor Synchronous Drive System

Where,

$$
\left\{\begin{array}{l}
\dot{F}_{1}=\frac{K_{1}}{T_{1}}\left(\frac{1}{n_{p 1}} r_{1} k_{1} w_{r 1}-\frac{1}{n_{p 2}} r_{2} k_{2} w_{r 2}\right)-\frac{F_{1}}{T_{1}} \\
\dot{F}_{2}=\frac{K_{2}}{T_{2}}\left(\frac{1}{n_{p 2}} r_{2} k_{2} w_{r 2}-\frac{1}{n_{p 3}} r_{3} k_{3} w_{r 3}\right)-\frac{F_{2}}{T_{2}}
\end{array}\right.
$$

$\omega_{r 1}, \omega_{r 2}, \omega_{r 3}: 1 \#$ motor speed(r/min), 2\#motor speed(r/min), 3\#motor speed(r/min)

$r_{1}, r_{2}, r_{3}: 1 \#$ roll $\operatorname{radius}(\mathrm{m}), 2 \#$ roll $\operatorname{radius}(\mathrm{m}), 3 \#$ roll $\operatorname{radius}(\mathrm{m})$

$k_{1}, k_{2}, k_{3}$ : speed ratio of $1 \#$ roll, speed ratio of 2\#roll, speed ratio of $3 \#$ roll

$n_{P 1}, n_{P 2}, n_{P 3}: 1 \#$ motor rotor poles, $2 \#$ motor rotor poles, 3\#motor rotor poles

$\mathrm{T} 1, \mathrm{~T} 2$ : time constant of tension variation

$\mathrm{K}_{1}, \mathrm{~K}_{2}$ : transfer coefficient

Laplace transform to Eq. (1) and simplify them as follows :

$$
\left\{\begin{array}{l}
F_{1}(s)=\left(\frac{1}{n_{p 1}} r_{1} k_{1} \omega_{r 1}-\frac{1}{n_{p 2}} r_{2} k_{2} \omega_{r 2}\right) \frac{K_{1}}{T_{1} s+1} \\
F_{2}(s)=\left(\frac{1}{n_{p 2}} r_{2} k_{2} \omega_{r 2}-\frac{1}{n_{p 3}} r_{3} k_{3} \omega_{r 3}\right) \frac{K_{2}}{T_{2} s+1}
\end{array}\right.
$$

If the motor speed and belt tension are regarded as controlled variables and the given of three inverters as input variables, then open loop control diagram of multi induction motor synchronous drive system can be obtained in Figure 2.

From the above analysis, there is coupling effect among 1\#motor speed $\omega_{r 1}$, the tension $F_{1}$ and $F_{2}$, and the affect mutually. Whenever the motor speed or belt tension changes, it will affect the other. Therefore, if we want to realize the synchronous control of the three motors, the decoupling of motor speed and belt tension is needed. 


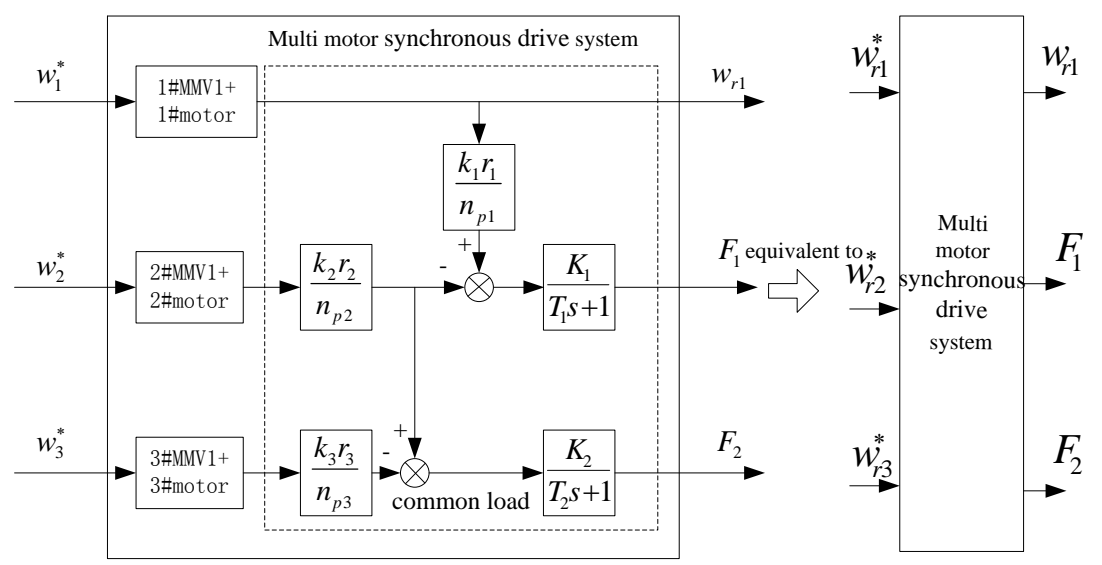

Figure 2. Open Loop Control Diagram of Multi Motor Synchronous Drive System

\section{Closed Loop Control of Multi Induction Motor Synchronous Drive System}

In order to further improve control performances of multi motor synchronous drive system, neural network control technology is used to design the control system. In order to eliminate the coupling effect between speed and tension loop, neuron decoupling and self-tuning PID control method is adopted to design a new control system. The schematic diagram of control system is shown in Figure.3. The structure is used the series open loop decoupling strategy that the neuron decoupling compensator set behind the self-tuning PID controller [7].

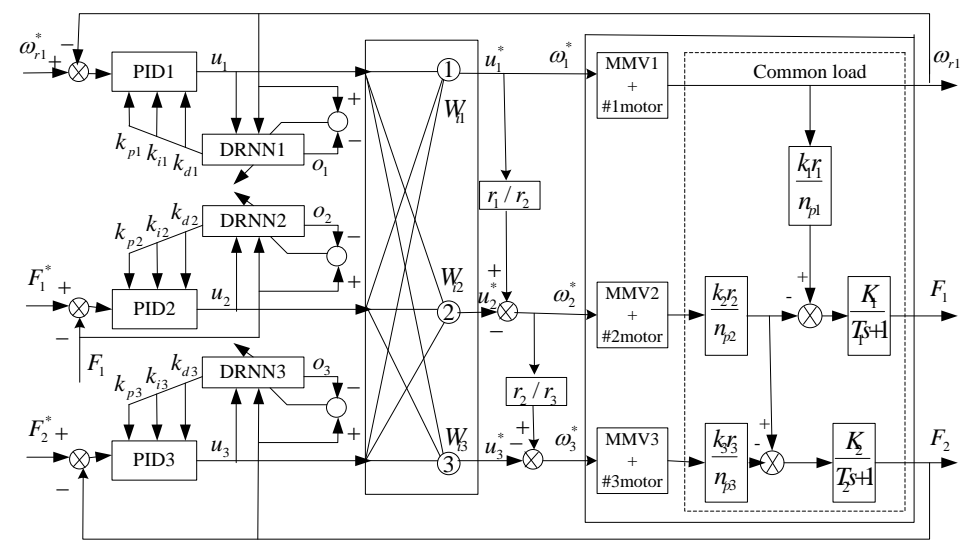

Figure 3. Control Diagram of Multi Motor Synchronous System

\subsection{Self-Tuning PID Controller of Speed and Tension Loop}

Self-tuning PID controllers based on DRNN are adopted in both speed and tension loops. DRNN is used as on-line identifier. According to the varying environment, It adjusts network weights automatically, and traces the output of controlled object. Jacobian information obtained by DRNN can be used to adjust the parameters of PID controller on-line. This controller has the advantages of fast response, strong adaptability and anti-interference. The principle of self-learning PID controller based on DRNN network is as follows: 
Position type PID controller is adopted in this paper. The object is controlled directly in closed loop, and $k_{P}, k_{i}$ and $k_{d}$ are online adjusted [8].

Control algorithm is as follows

$$
\begin{aligned}
& \operatorname{error}(k)=r(k)-y(k) \\
& \left\{\begin{array}{l}
x_{1}(k)=\operatorname{error}(k) \\
x_{2}(k)=\sum_{i=1}^{k}(\operatorname{error}(k) \times T) \\
x_{3}(k)=\frac{\operatorname{error}(k)-\operatorname{error}(k-1)}{T} \\
u(k)=k_{p}(k) x_{1}(k)+k_{i}(k) x_{2}(k)+k_{d}(k) x_{3}(k)
\end{array}\right.
\end{aligned}
$$

Where,

$r(k)$ : given speed $\omega_{r 1} *$ or input tension $F^{*}$

$y(k)$ : actual output speed $\omega_{r 1}$ or output tension $f$

$T$ : sampling time

Index function can be defined as follows:

$$
E(k)=\frac{1}{2}(r(k)-y(k))^{2}=\frac{1}{2} e(k)^{2}
$$

Gradient descent method is adopted to adjust the parameters of $k_{p}, k_{i}$ and $k_{d}$

$$
\left\{\begin{array}{l}
E(k)=\frac{1}{2}(r(k)-y(k))^{2} \\
\Delta k_{p}=-\eta_{p} \frac{\partial E}{\partial k_{p}}=-\eta_{p} \frac{\partial E}{\partial y} \frac{\partial y}{\partial u} \frac{\partial u}{\partial k_{p}}=\eta_{p} e(k) \frac{\partial y}{\partial u} x_{1}(k) \\
\Delta k_{i}=-\eta_{i} \frac{\partial E}{\partial k_{i}}=-\eta_{i} \frac{\partial E}{\partial y} \frac{\partial y}{\partial u} \frac{\partial u}{\partial k_{i}}=\eta_{i} e(k) \frac{\partial y}{\partial u} x_{2}(k) \\
\Delta k_{d}=-\eta_{d} \frac{\partial E}{\partial k_{d}}=-\eta_{d} \frac{\partial E}{\partial y} \frac{\partial y}{\partial u} \frac{\partial u}{\partial k_{d}}=\eta_{d} e(k) \frac{\partial y}{\partial u} x_{3}(k)
\end{array}\right.
$$

$\frac{\partial y}{\partial u}$ is the Jacobian information of controlled object, which can be obtained by identification of DRNN.

DRNN is a recurrent neural network. It is made of three layers network structure, whose hidden layer is recurrent layer. The 3-7-1 network structure is shown in Figure 4.

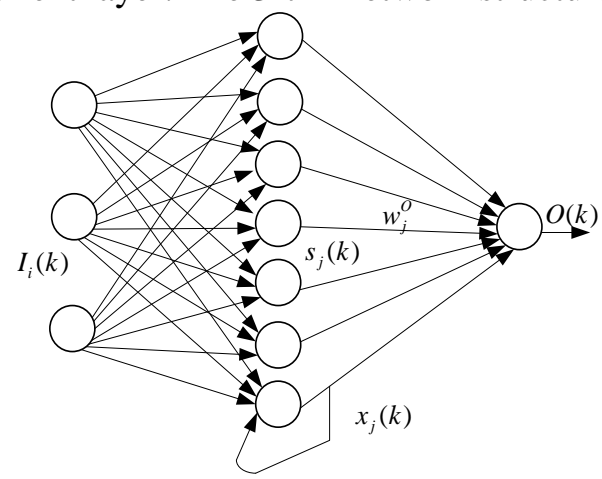

Figure 4. The Structure of DRNN 
In DRNN network, $I=\left[I_{1}, I_{2}, \ldots, I_{n}\right]$ is set as input vector, and $I_{i}(k)$ is set as the ith neuron input. In this paper, the input vector of the network is $I=\{u(k-1), y(k), 1.0\}$.The algorithm of DRNN is

$$
\left\{\begin{array}{l}
y_{m}(k)=O(k)=\sum_{j} W_{j}^{o} X_{j}(k) \\
X_{j}(k)=f\left(S_{j}(k)\right) \\
S_{j}(k)=W_{j}^{D} X_{j}(k-1)+\sum_{i} W_{i j}^{I} I_{i}(k)
\end{array}\right.
$$

Where

$X_{j}(k)$ : the jth neuron output of recurrent layer

$S_{j}(k):$ the jth recurrent neuron input sum

$f(\cdot)$ : double $\mathrm{S}$ function

$O(k), y_{m}(k)$ : output of DRNN

$W^{D}$ : weight vector of recurrent layer

$W^{O}$ : weight vector of output layer

$W^{I}$ : weight vector of input layer

The identification error is

$$
e m(k)=y(k)-y m(k)
$$

The identification index is

$$
E m(k)=\frac{1}{2} e m(k)^{2}
$$

Learning algorithm adopts gradient descent method

$$
\left\{\begin{array}{l}
\Delta w_{j}^{O}(k)=-\frac{\partial E m(k)}{\partial w_{j}^{O}}=e m(k) \frac{\partial y m}{\partial w_{j}^{O}}=e m(k) X_{j}(k) \\
w_{j}^{O}(k)=w_{j}^{O}(k-1)+\eta_{o} \Delta w_{j}^{O}(k)+\alpha\left(w_{j}^{O}(k-1)-w_{j}^{O}(k-2)\right) \\
\Delta w_{i j}^{I}(k)=-\frac{\partial E m(k)}{\partial w_{i j}^{I}}=e m(k) \frac{\partial y m}{\partial X_{j}} \frac{\partial X_{j}}{\partial w_{i j}^{I}}=e m(k) w_{j}^{O} Q_{i j}(k) \\
w_{i j}^{I}(k)=w_{i j}^{I}(k-1)+\eta_{I} \Delta w_{i j}^{I}(k)+\alpha\left(w_{i j}^{I}(k-1)-w_{i j}^{I}(k-2)\right) \\
\Delta w_{j}^{D}(k)=-\frac{\partial E m(k)}{\partial w_{j}^{D}}=e m(k) \frac{\partial y m}{\partial X_{j}} \frac{\partial X_{j}}{\partial w_{j}^{D}}=e m(k) w_{j}^{O} P_{j}(k) \\
w_{j}^{D}(k)=w_{j}^{D}(k-1)+\eta_{D} \Delta w_{j}^{D}(k)+\alpha\left(w_{j}^{D}(k-1)-w_{j}^{D}(k-2)\right)
\end{array}\right.
$$

Double $\mathrm{S}$ function of recurrent layer neuron is

Where

$$
\begin{gathered}
f(x)=\frac{1-e^{-x}}{1+e^{-x}} \\
P_{j}(k)=\frac{\partial X_{j}}{\partial w_{j}^{D}}=f^{\prime}\left(S_{j}\right) X_{j}(k-1) \\
Q_{i j}(k)=\frac{\partial X_{j}}{\partial w_{i j}^{I}}=f^{\prime}\left(S_{j}\right) I_{i}(k)
\end{gathered}
$$

$\eta_{I}$ : learning rate of input layer

$\eta_{D}$ : learning rate of recurrent layer

$\eta_{O}$ : learning rate of output layer 


$$
\alpha \text { : inertial coefficient }
$$

Jacobian information of the controlled object $\frac{\partial y}{\partial u}$ is

$$
\frac{\partial y}{\partial u} \approx \frac{\partial y m}{\partial u}=\sum_{j} w_{j}^{o} f^{\prime}\left(S_{j}\right) w_{i j}^{I}
$$

\subsection{Adaptive Neuron Decoupling Compensator of Multi Variable System}

Neuron decoupling compensator views the coupling effect from other channels as measurable disturbances to compensate. It makes the generalized system composed of neuron decoupling compensator and multi motor synchronous drive system into the system without or with a little coupling, by adjusting weights of neuron compensation network. The serial open loop decoupling strategy is adopted in this paper, that is, decoupling compensator is set behind controller. A single neuron is set in every pair input and output channel. Each single neuron has three inputs, and each of them accept control signal $u_{1}, u_{2}$ and $u_{3}$ of the three self-tuning PID controllers. The output $u^{*}$ of each single neuron is used as control signal, which is compensated, to feed multi variable system. The decoupling of the speed and tension is finally realized by correcting weights of neuron [9].

The algorithm of neuron decoupling compensator in Figure. 3 is:

$$
\left\{\begin{array}{l}
u_{j}^{*}(k)=\sum_{j=1}^{3} w_{i j} u_{j}(k) \\
w_{i j}(k+1)=w_{i j}(k)+\eta\left(\bar{u}_{i}^{*}(k)-u_{i}^{*}(k)\right) u_{j}(k)
\end{array}\right.
$$

The initial value of weight $w_{i j}$ is selected as $\left\{\begin{array}{l}w_{i j}=1(i=j) \\ w_{i j}=0(i \neq j)\end{array}\right.$, which equals to without decoupling status. $\eta$ is learning rate. $u_{j}^{*}(k)$ is actual output and $\bar{u}_{i}^{*}(k)$ is expected output of neuron.

$J_{i}=\frac{1}{2}\left(r_{i}(k)-y_{i}(k)\right)^{2}$ is regarded as the objective function. In order to make $J_{i}$ minimum, gradient descent method is adopted to search, so the calculation formula of weight coefficient is:

$$
\left\{\begin{array}{l}
w_{i j}(k+1)=w_{i j}(k)+\eta\left(r_{i}(k)-y_{i}(k)\right) \operatorname{sgn} \frac{y_{i}(k+1)-y_{i}(k)}{u_{i}^{*}(k)-u_{i}^{*}(k-1)} u_{j}(k)(i \neq j) \\
w_{i j}=1 \quad(i=j)
\end{array}\right.
$$

\section{Experiment Results of the Multi Induction Motor Synchronous Drive System}

\subsection{Introduction of the Experimental Hardware System}

The experiments are carried out on multi induction motor synchronous control equipment developed by ourselves [5] [10]. The experimental platform are mainly composed of common load, three MMV inverters, three AC motors of $380 \mathrm{~V} / 50 \mathrm{~Hz} / 2.2 \mathrm{~kW}$, upper computer, S7-300 PLC, photoelectric encoder, tension sensor and other equipments. Figure.5 is the structural diagram of multi motor synchronous experimental platform. 


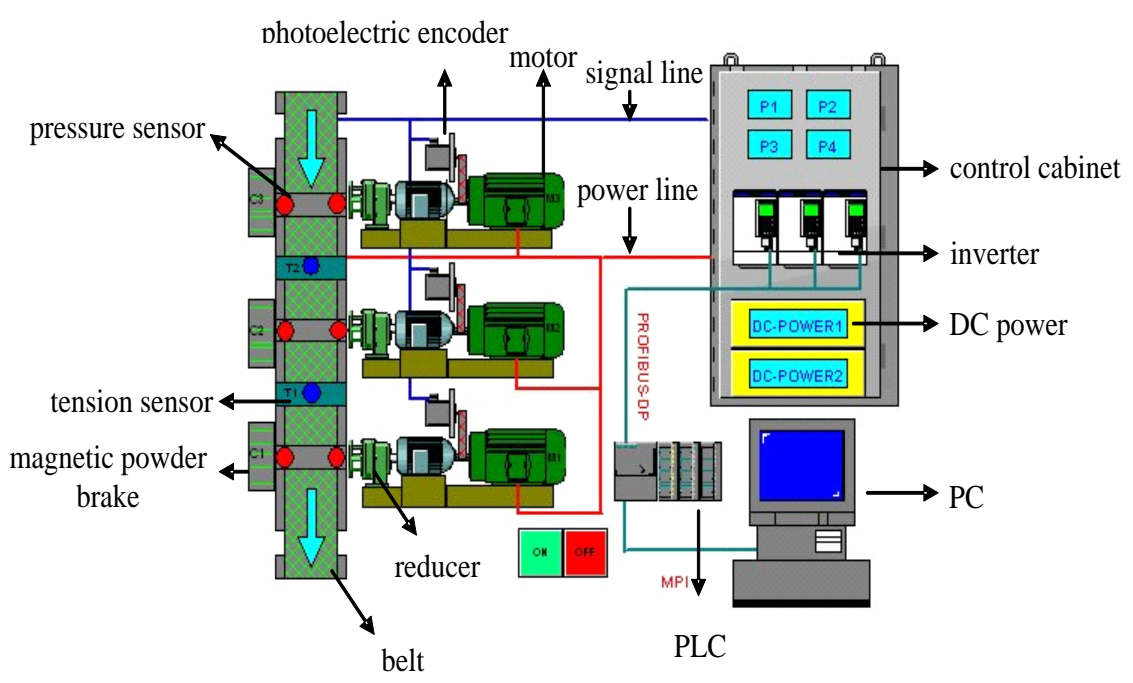

Figure 5. Multi Motor Synchronous Drive System Experimental Platform

\subsection{PLC Hardware Configuration}

A 315-2DP is selected as the CPU of S7-300PLC. It has MPI and Profibus-DP communication ports. It also has medium and large scale program storage capacity and data structure, and strong treatment ability to floating-point operation. Therefore, it can meet the requirements of neural network control algorithm, which has a lot of floatingpoint operation. A power module PS307 10A, supplied by ac 220V, provides four-channel $24 \mathrm{~V}$ dc power supply. A digital input module (DI16 $\times$ DC24V, Alarm) realizes switch signal start-stop control. A analog input/output module $(\mathrm{AI} 4 / \mathrm{AO} 4 \times 14 / 12 \mathrm{Bit})$ realizes tension signals collection. A high speed counter module FM350-1 realizes the count of pulses emitted by photoelectric encoder. The main hardware type in the PLC control system are shown in Table 1.

\section{Table 1. Main Hardware Type of PLC Control System}

\begin{tabular}{ccc}
\hline name & type & quantity \\
\hline CPU & 6ES7 307-1KA00-0AA0 & 1 \\
Power supply & 6ES7 315-2AG10-0AB0 & 1 \\
Memory card & 6ES7 953-81M20-0AA0 & 1 \\
digital input & 6ES7 321-7BH01-0AB0 & 1 \\
analog input/output & 6ES7 335-7HG01-0AB0 & 1 \\
counter & 6ES7 350-1AH03-0AE0 & 1 \\
frame & 6ES7 390-1AF30-0AA0 & 1 \\
inverter & 6SE9221-0BC40 & 3 \\
\hline
\end{tabular}

\subsection{Parameters Measurement}

\subsubsection{Speed Sample}

Siemens high speed counter module FM350-1 is adopted to count the pulses emitted by photoelectric encoder. In this experimental system, the type of photoelectric encoder is YGM-615, whose working voltage is $5 \mathrm{~V} \pm 5 \% .2048$ pulses are emitted by photoelectric encoder during every round. $M$ count method is adopted in the experiment. Then, the calculation formula of speed and electric angular velocity can be obtained as follows: 


$$
\begin{aligned}
& \omega=\frac{2 \pi \times \frac{c}{e}}{T} \\
& n=\frac{60 \times \omega}{2 \pi}=\frac{60 \times \frac{c}{e}}{T}
\end{aligned}
$$

Where,

$n$ : the speed of motor $(r / \min )$,

$\omega$ : electric angular velocity $(\mathrm{rad} / \mathrm{s})$,

$T:$ count period, $T=0.1 \mathrm{~s}$,

$c$ : pulse count value of high speed counter module,

$e:$ pulse number emitted by photoelectric encoder during every round.

Then, the final calculation formula of speed is:

$$
n=0.29296875 \times c \quad(r / \mathrm{min})
$$

\subsubsection{The Detection of Tension Displacement}

Two sets of SL100 sensor are adopted to detect the belt tension between the two adjacent motors, whose working voltage is $12 \mathrm{~V}$, output voltage is $1 \sim 5 \mathrm{~V}$, and range is $50 \mathrm{~kg}$. Concrete calibration data are shown in Table 2, and the mathematical relation can

\begin{tabular}{|c|c|c|c|c|c|c|c|}
\hline range & $\begin{array}{l}0.0 \\
0\end{array}$ & 10 & 20 & 30 & 40 & 50 & $\mathrm{Kg}$ \\
\hline \multirow[t]{2}{*}{ progress } & 1.0 & 1.8 & 2.5 & 3.4 & 4.2 & 5.0 & \multirow{2}{*}{$\mathrm{V}$} \\
\hline & 00 & 03 & 96 & 00 & 01 & 01 & \\
\hline \multirow[t]{2}{*}{ return } & 1.0 & 1.8 & 2.5 & 3.4 & 4.2 & 5.0 & \multirow{2}{*}{ V } \\
\hline & 00 & 01 & 96 & 00 & 00 & 00 & \\
\hline \multirow[t]{2}{*}{ standard value } & 1.0 & 1.8 & 2.5 & 3.4 & 4.2 & 5.0 & \multirow{2}{*}{$\mathrm{Kg}$} \\
\hline & 00 & 00 & 96 & 00 & 00 & 00 & \\
\hline
\end{tabular}
be expressed approximately shown in Figure 6.

Table 2. Tension-Voltage Calibration Data Table

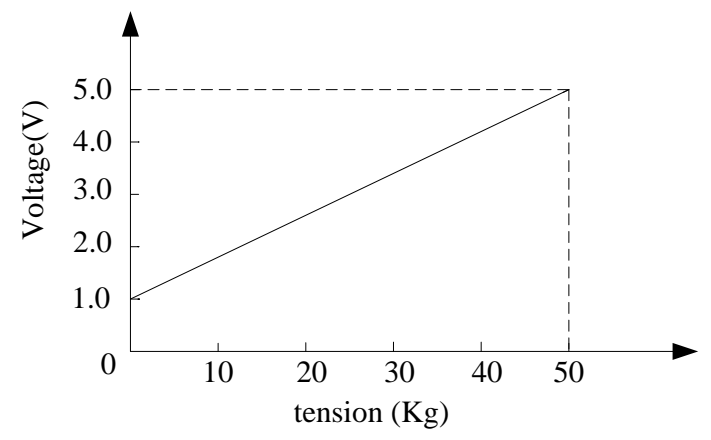

Figure 6. Tension-Voltage Calibration Chart

\subsection{The Design of Structured Program}

There are three programming methods in STEP7, linear, modularization and structured programming. The third method is adopted in this paper. A complex automation task is usually deposed into small task blocks (FC or FB) which are able to reflect process technology and function, or reusable, then OB1 is used to call these task blocks. This programming idea is in good agreement with design requirement of the subject programming, so structured programming is introduced. The program structure diagram 
of the multi motor synchronous drive system is shown in Figure 7. Ladder diagram programming is adopted in industrial software STEP7 V5.4. The software of control system is designed with structural program language, and the program flowcharts are shown in Figure 8 and Figure 9.

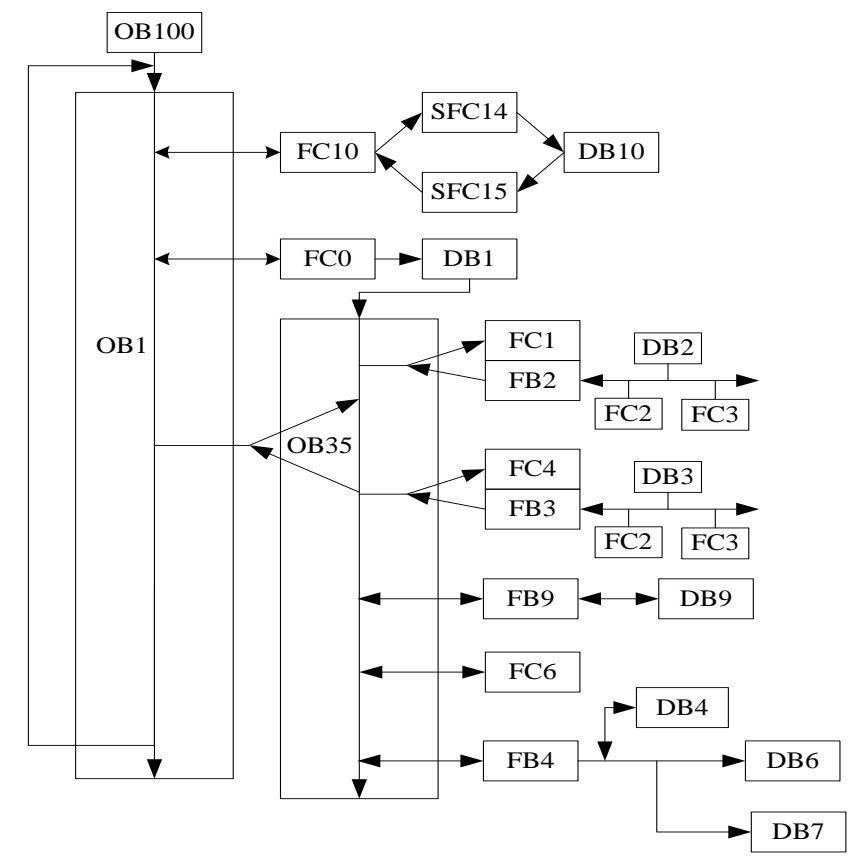

Figure 7. Program Structure Diagram of the Multi Induction Motor System

The main task blocks in Figure 7 are listed in Table.3.

Table 3. Blocks in the Project

\begin{tabular}{cc}
\hline Block & Function description \\
\hline OB1 & main program circulation \\
OB35 & circulation interruption, used in process PID \\
OB100 & warm start \\
FC0 & system function of high speed counter \\
FC1,FC4 & set induction motor speed and belt tension \\
FC6 & convert speed into frequency \\
FC10 & inverter communication \\
FB2, FB3 & self-tuning PID based on DRNN algorithm \\
FB4 & file speed and tension sampling data \\
FB9 & neuron decoupling compensation algorithm \\
DB1 & sharing data block of FC0 \\
DB2, DB3, DB4, & instance data block of FB2, FB3, FB4, FB9, \\
DB9 & sharing data block for saving actual speed and \\
DB6,DB7 & tension \\
DB10 & sharing data block for inverter communication \\
UDT1 & user defined data block of high speed counter \\
SFC14 & read data from Profibus slave station \\
SFC15 & write data to Profibus slave station \\
\hline
\end{tabular}




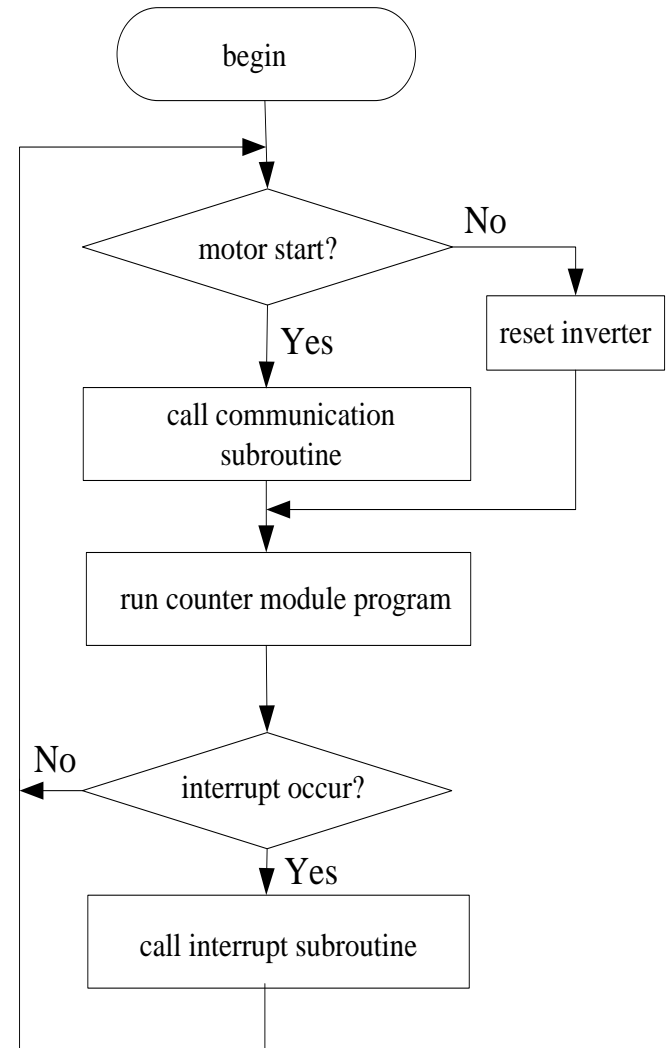

Figure 8. The Flowchart of OB1

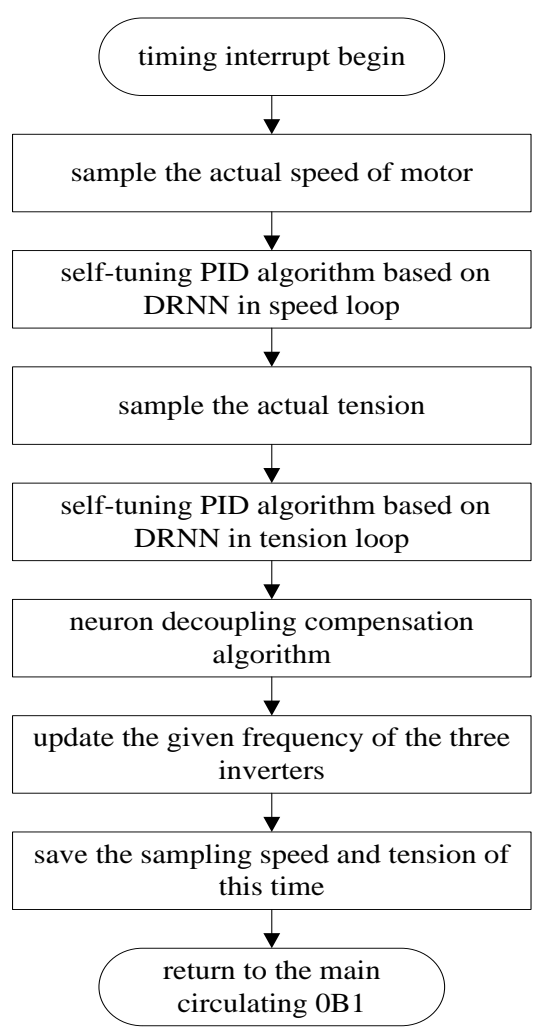

Figure 9. The Flowchart of OB35

\section{Experiment Wave and Its Analysis}

\subsection{Load Experiment}

At first, the speed of $1 \#$ motor $\omega_{r 1}^{*}$ is given $150 \mathrm{r} / \mathrm{min}, \quad F_{1}^{*}$ is given $10 \mathrm{~kg}$ and $F_{2}^{*}$ is given $12 \mathrm{~kg}$. After free-load start, load increases at 80 second and decreases at 120 second, speed and tension response curves of multi motor system are shown in Figure.10. As can be seen, neuron network control can make corresponding control adjustment according to load changes, so as to keep speed and tension constant furthest with no influence of load changes.

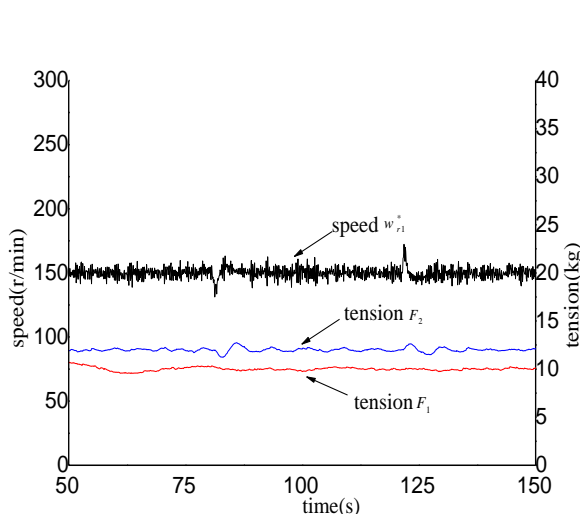

(a) PID control

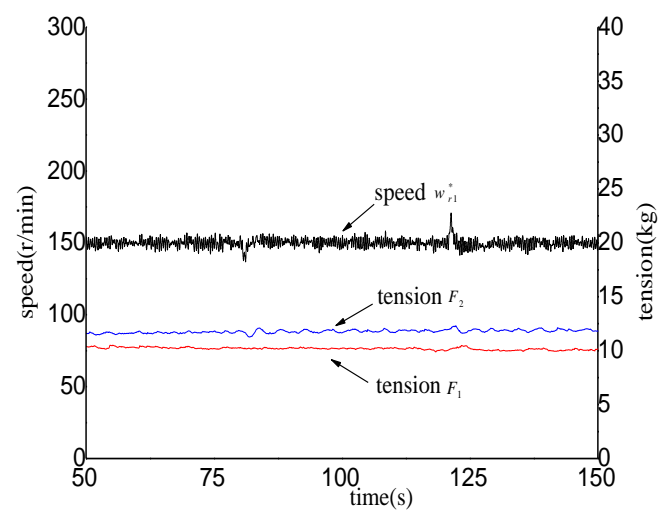

(b) neuron network control

Figure 10. The Response of Sudden Load Change 


\subsection{Decoupling Experiment}

Decoupling effect is the main index to measure the control scheme of multi motor synchronous drive system. Taking the system response of sudden speed changes as example, the decoupling effect with PID control and neuron network control are compared. At first, the speed of $1 \#$ motor $\omega_{r 1}^{*}$ is given $300 \mathrm{r} / \mathrm{min}, F_{1}^{*}$ is given $10 \mathrm{~kg}$ and $F_{2}^{*}$ is given $12 \mathrm{~kg}$. After free-load start, the given speed $\omega_{r 1}^{*}$ increases or decreases at 100 second, experiment results are shown in Figure.11 and Figure.12. As can be seen, the better decoupling control of speed and tension is realized with neural network control method. In contrast to PID control, it can reduce the influence of speed variation on tension.
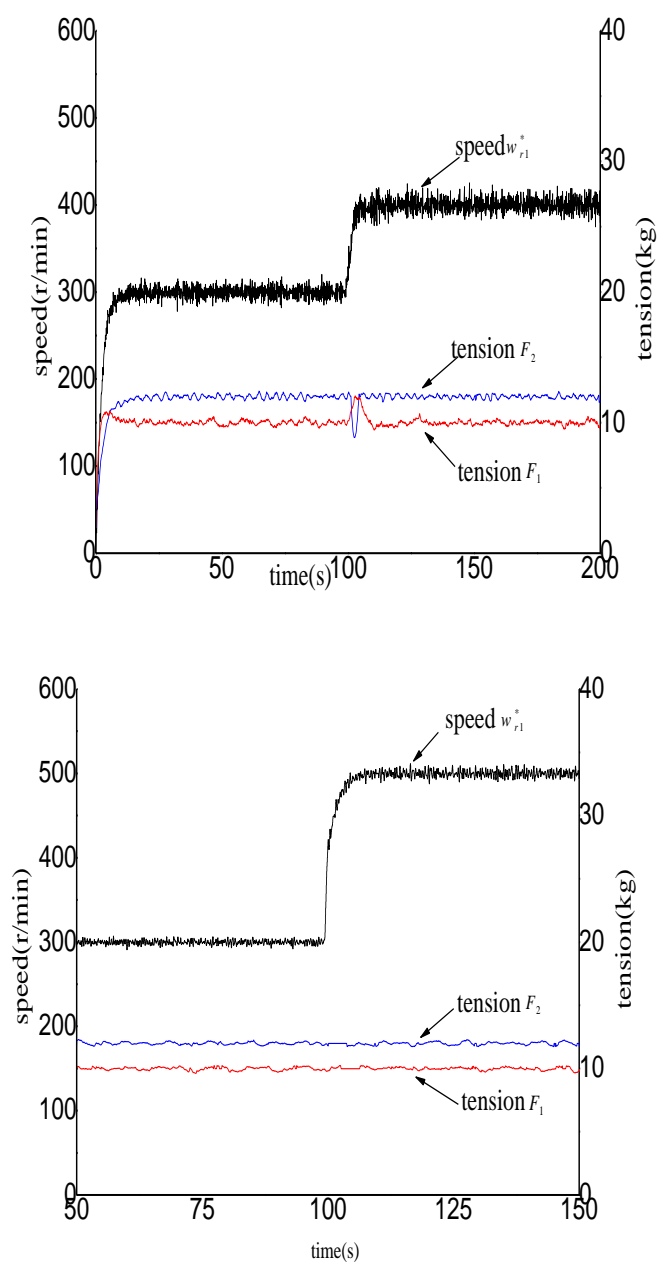

(a) PID control

(b) neuron network control

Figure 11. The Response of Speed Sudden Increase with Constant Tension 

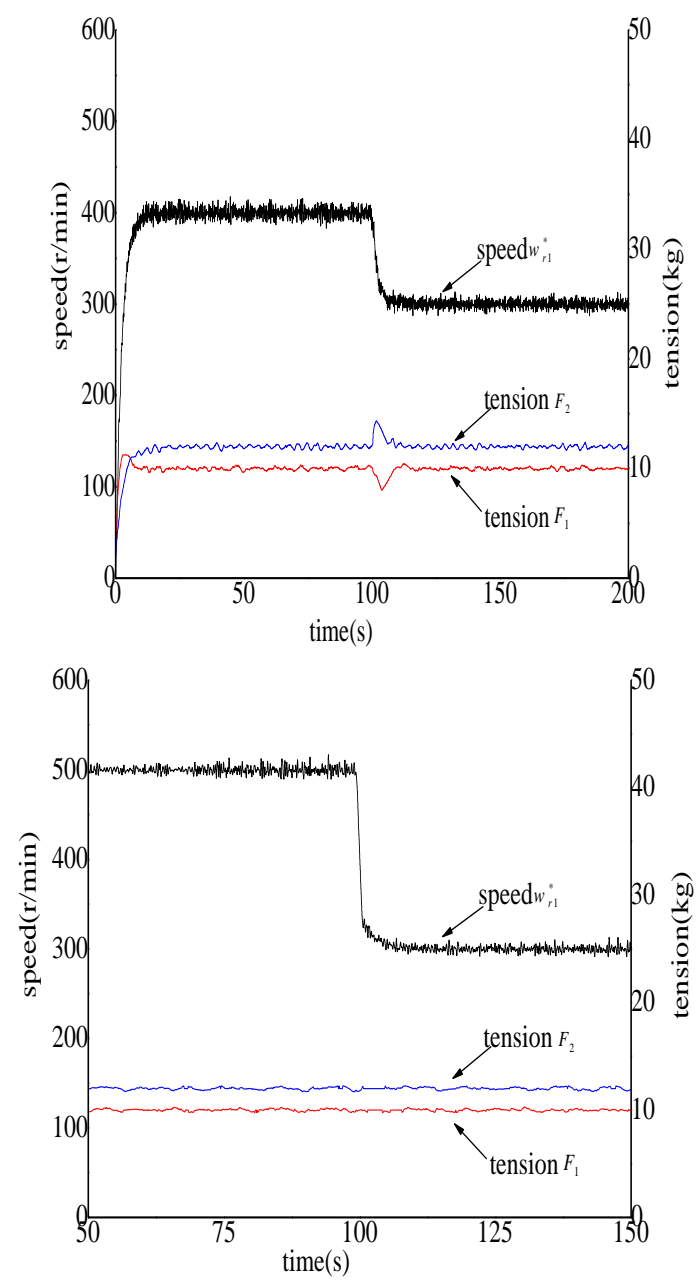

(a) PID control

(b) neuron network control

Figure 12. The Response of Speed Sudden Decrease with Constant Tension

\subsection{Tracking Experiment}

At first, the speed of $1 \#$ motor $\omega_{r 1}^{*}$ is given $300 \mathrm{r} / \mathrm{min}$, and it varies from $300 \mathrm{r} / \mathrm{min}$ to $600 \mathrm{r} / \mathrm{min}$ in the form of triangular wave after 40 second. The response of speed tracking triangular curves are shown in Figure.13. And the response of speed tracking single period triangular curves are shown in Figure.14. As can be seen from Figure.13(a) and Figure.14(a), the response to the output speed of 1\#motor are following triangle waves, appearing a certain error, but in Figure.13(b) and Figure.14(b), the error of both almost is zero. 

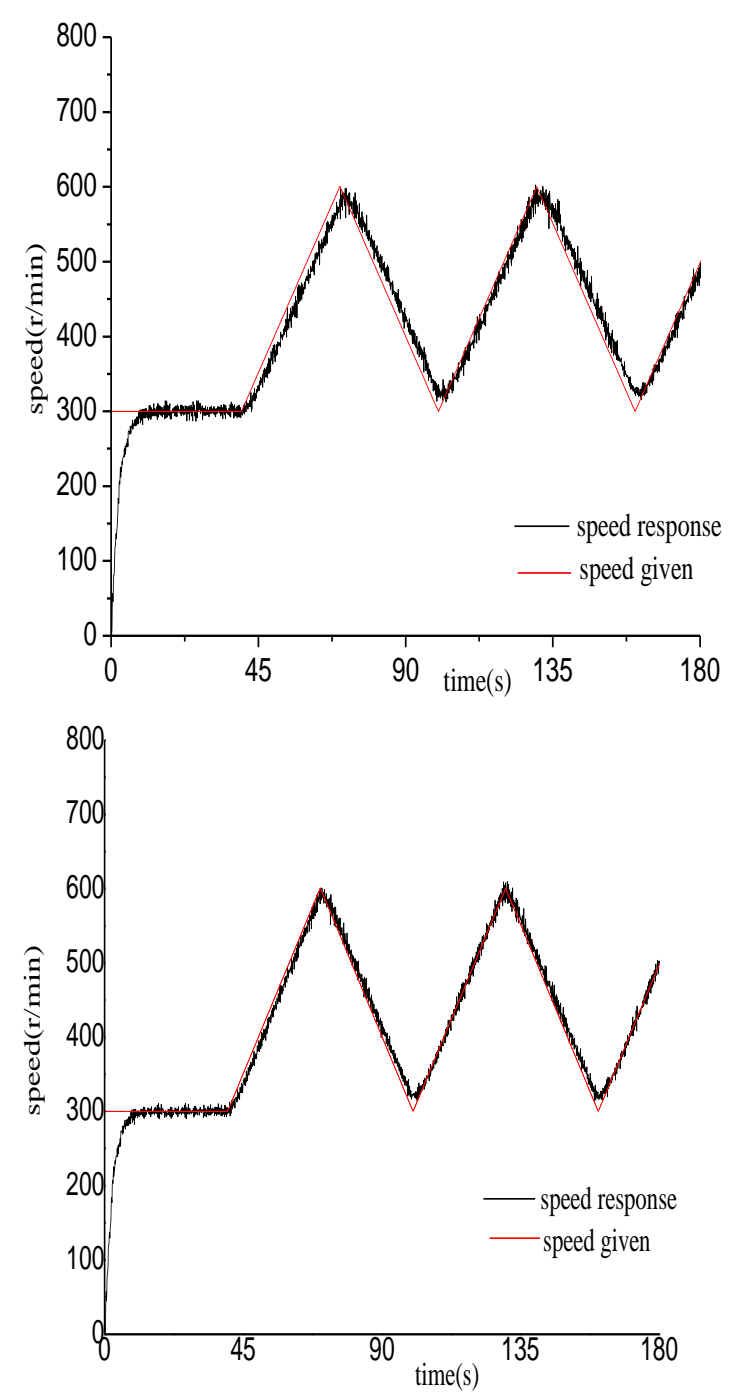
(a) PID control
(b) neuron network control

Figure 13. The Response of Speed Tracking Triangular Curve

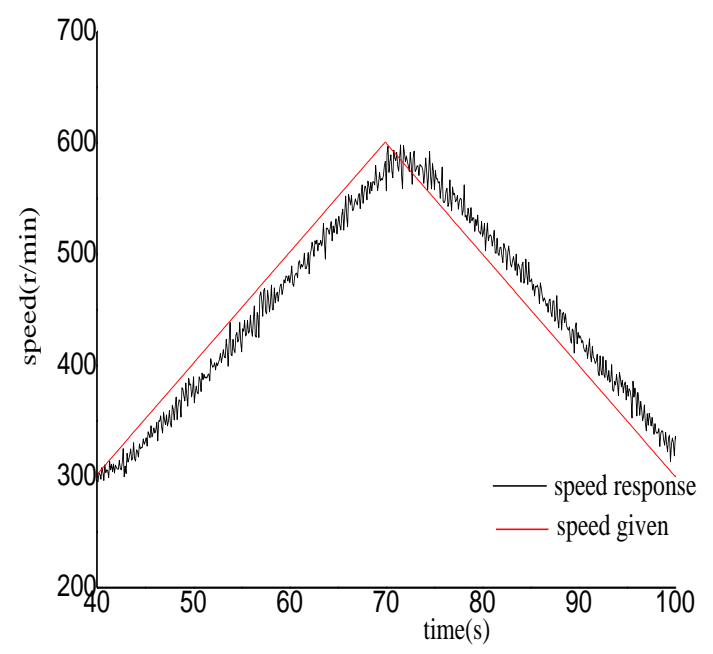




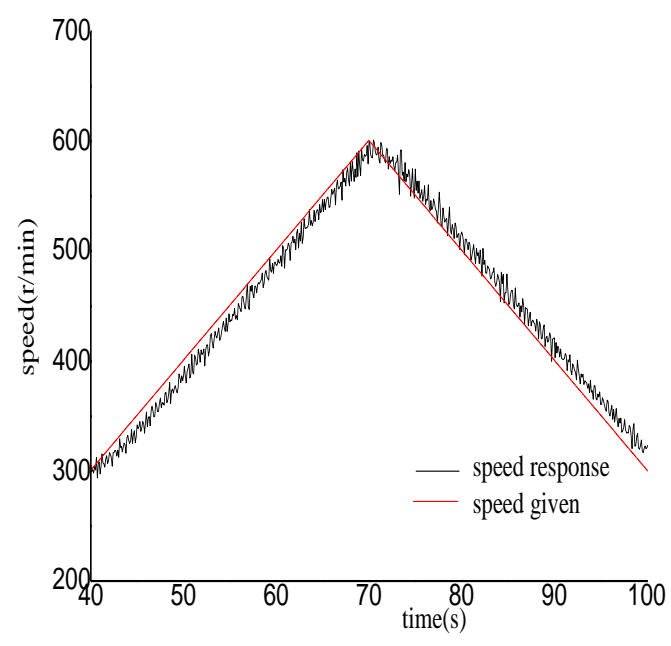

(a) PID control

(b) neuron network control

Figure 14. The Response of Speed Tracking Single Period Triangular Curve

At first, the speed of $1 \#$ motor $\omega_{r 1}^{*}$ is given $600 \mathrm{r} / \mathrm{min}$, and it varies from $600 \mathrm{r} / \mathrm{min}$ to $800 \mathrm{r} / \mathrm{min}$ in the form of square wave after 40 second. The response of speed tracking square curves and the local amplification curves are shown in Figure.15 and Figure.16. As can be seen from the curves, neural network control has faster response and higher steady precision.

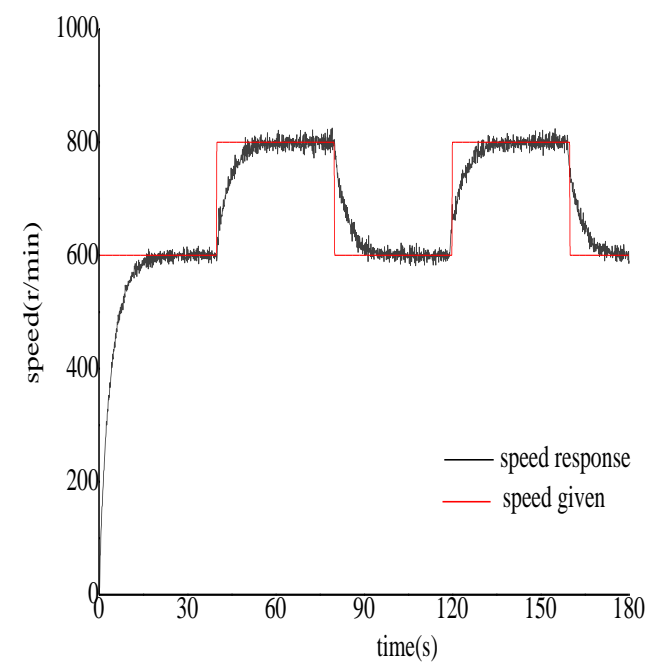




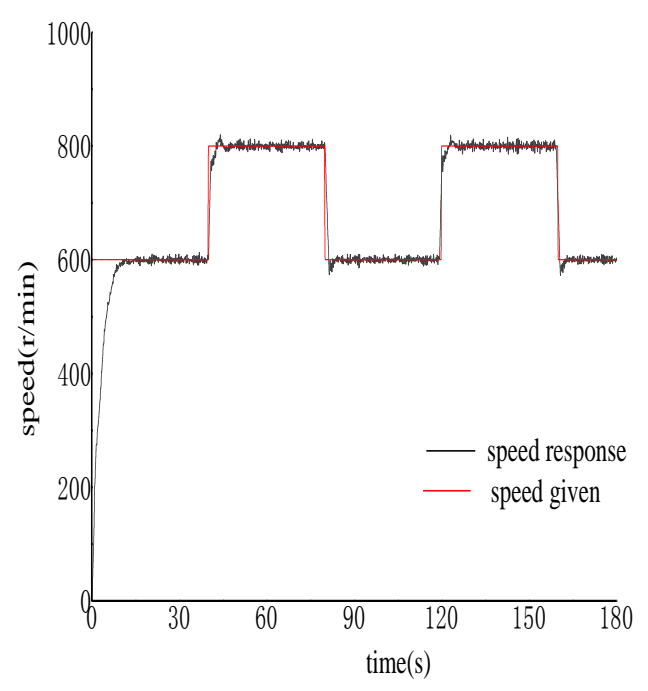
(a) PID control
(b) neuron network control

Figure 15. The Response of Speed Tracking Square Curve
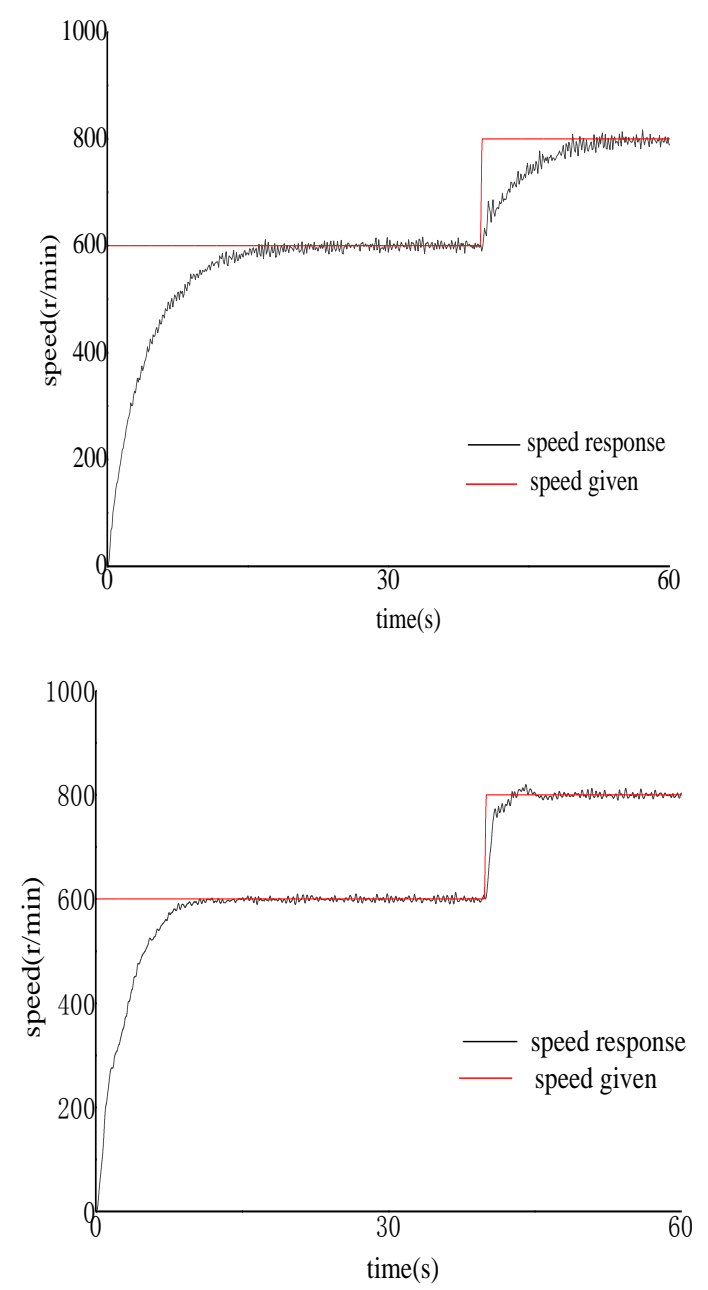

$\begin{array}{lll}\text { (a) PID control } & \text { (b) neuron network control }\end{array}$

Figure 16. The Response of Speed Tracking Square Local Amplification Curve 


\section{Conclusion}

This paper aiming at multi motor synchronous drive system which is multi variable, nonlinear, and coupling, presents a neural network compound controller composed of neuron decoupling compensator and self-tuning PID control method based on multivariable decoupling and intelligent control theory. It finally realizes the synchronous control of the system based on S7-300 PLC experiment platform. This method is proved to be applied into the synchronous control of three motor drive system, so a new control way of three motor system is presented. The project is further studied by increasing tension self-tuning PID controllers and the dimensions of neuron decoupling compensator, and the authors find that the method can be extended to the usual multi motor synchronous drive system $(n \geq 3)$, which can satisfy the requests of the actual production.

\section{Acknowledgements}

This work was supported in part by the National Natural Science Foundation of China under Grant 51507150 and 51407153.

\section{References}

[1] S. H. Song and S. K. Sul, "A new tension controller for continuous strip processing Line", IEEE Transactions on Industry Applications, vol.36, no.2, (2000), pp.633-639.

[2] H. Zhang and G. H. Liu, „Decoupling control of artificial neural network inverse system in AC variable frequenly induction motor system", Journal of Jiangsu University Natural Science Edition, vol.23, (2002), pp. 88-91.

[3] C. Chen, "Neural network control of induction motor speed control system", International Journal of Control and Automation, (2014), vol.7, no.10, pp.243-254.

[4] X. Z. Dai and G. H. Liu, "The neural network inverse control of the constant ratio of voltage and frequency adjusting speed system", Chinese Electrical Engineering College Journal,vol.25, no.7, (2005), pp.109-114.

[5] X.Q. Liu and L. Tang, "Three-motor synchronous control system based on fuzzy active disturbances rejection system", Electric machines and control, vol.17, (2013), pp.104-109.

[6] J. Z. Zhang and G. H. Liu, "Multi-model identification to multi-motor synchronous system", Machines and Control, vol. 13, no. 1, (2009), pp.138-142.

[7] P. Jiang, Z. J. Li and W. P. Liang, "A decoupling algorithm based on neuron", Journal of North China Electric Power University, vol.27, no.2, (2004), pp.47-51.

[8] Z. H. Cui, X. J Cai and J. C. Zeng, "PID-controlled particle swarm optimization", Journal of MultipleValued Logic and Soft Computing, vol.16, no.6, (2010), pp.585-610.

[9] L. Dong and Y. Bai, "Neural decoupling control for multi variable system", Modern Electric Power, vol.16, no.1, (1999), pp.11-15.

[10] C. Chen and G. W. Hu, "Fuzzy PID control of induction motor speed regulating system", International Journal of Wireless and Mobile Computing, vol. 6, no. 4, (2013), pp.321-330.

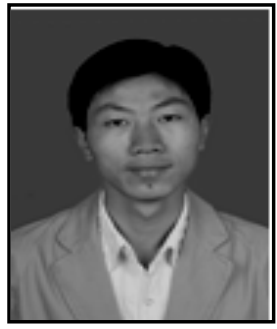

\section{Authors}

Chong Chen, he was born in Jiangsu Province, China, in 1982. He received his master degree in Electrical Engineering from Jiangsu University in 2008. He is currently working at School of Electrical Engineering, YanCheng Institute of Technology, China. His current research interest include motion control and power electron application. 

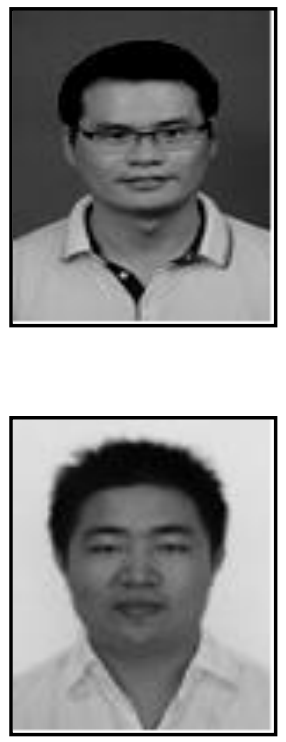

Simin Peng, he was born in Hunan province, China, in 1980. $\mathrm{He}$ received the B.S degree in automation from Xiangtan University, Xiangtan, China, in 2003, and M.S. degree in 2008, and Ph.D degree in electrical engineering from Shanghai Jiao Tong University in 2013. He is currently working at Yancheng Institute of Technology. His research interests include wind power, microgrid, battery energy storage system and battery manage system.

Zhilei Yao, he was born in Jiangsu Province, China, in 1981. $\mathrm{He}$ received the B.S., M.S., and Ph.D. degrees in electrical engineering from Nanjing University of Aeronautics and Astronautics, Nanjing, China, in 2003, 2006, and 2012, respectively.He is with the School of Electrical Engineering, Yancheng Institute of Technology, Yancheng, China, where he is currently an Associate Professor. He holds fourteen patents, and is the author or coauthor of more than 60 technical papers. His current research interests include $\mathrm{dc}-\mathrm{dc}$ converters, inverters, and distributed power generation 
International Journal of Control and Automation Vol. 9, No. 10 (2016) 